\title{
ENTRE EL HOGAR Y EL TRABAJO. MUJERES ASALARIADAS EN LA AGRICULTURA DEL VALLE DE UCO, PROVINCIA DE MENDOZA, ARGENTINA.
}

\author{
Elena Mingo \\ CEIL-PIETTE CONICET, Buenos Aires, Argentina
}

\begin{abstract}
Resumen.- En este artículo se analiza la participación de las mujeres en el trabajo asalariado agrícola en el Valle de Uco en la provincia de Mendoza, Argentina, a partir de su articulación con el trabajo doméstico y considerando las características de los hogares de las trabajadoras.En este sentido, se estudia la vinculación entre las trayectorias vitales y las inserciones laborales de las trabajadoras con el objetivo de comprender de qué forma las diferentes etapas del ciclo vital modifican las posibilidades de inserción laboral y, además, la manera en que las mujeres despliegan una diversidad de estrategias para compatibilizar el trabajo asalariado con el trabajo doméstico y reproductivo. Los avances de investigación que se presentan en este artículo son producto de tres etapas de trabajo de campo en la región que han comprendido la realización de entrevistas a empleadores y a trabajadoras asalariadas utilizando la historia de vida para reconstruir sus trayectorias laborales en el contexto de las etapas del ciclo vital atravesadas por las trabajadoras.
\end{abstract}

Palabras clave.- Trabajo, agricultura, género, mujeres.

Abstract.- This article analyze female wageworkers participation in agricultural sector of Uco's Valley, in Mendoza, Argentine, considering his linkages with house work and with households female wageworker's features. To follow this objective, I study relationship between wageworker life's histories and their labor paths, to understand the way in which different life's stages modify their possibilities of getting a job and the way in which women develop several strategies to make compatible wage work with house work and reproduction work. This article is part of research's advances emerged from three fieldwork's stages in Uco's Valley region, which consisted in making interviews to employers and female wageworkers life's histories to know their labor paths related to their life's stages.

Keywords.- Work, agriculture, gender, women.

\section{Introducción}

La creciente participación de las mujeres en el trabajo asalariado es uno de los fenómenos por los que más se han interesado los estudios sobre las transformaciones en las relaciones sociales de género en los últimos años. En este sentido, una de las preocupaciones más difundidas ha sido indagar acerca de la forma en que las trabajadoras articulan su participación en el mercado laboral y en el trabajo del hogar. El aumento de la participación de las mujeres en el mercado de trabajo no ha sido acompañado de un cambio en las relaciones de género que disminuya su carga de trabajo en el hogar, por lo que es importante destacar las implicancias que tiene esta doble presencia y las diferentes estrategias desarrolladas por las mujeres para desempeñarse en ambos ámbitos. 
Tanto en la Argentina como en Latinoamérica, el aumento de la participación femenina en la fuerza de trabajo se produjo en el contexto de la aplicación de políticas de estabilización y ajuste estructural que contribuyeron a la precarización del empleo afectando a los trabajadores de ambos sexos. Los estudios sobre el tema han señalado que la aplicación de políticas neoliberales no ha sido neutral en relación con el género, ya que, al desempeñar los hombres y las mujeres roles diferentes en la producción y reproducción, las políticas estatales no los afectan de igual manera. Asimismo, han destacado que la transferencia de los costos de reproducción de la fuerza laboral desde el Estado a los hogares, como resultado de dichas políticas, dificultó el acceso de los trabajadores a servicios sociales básicos antes provistos por el sistema de protección laboral. (Deere, 2006)

Esta nueva realidad impuso mayores exigencias a las mujeres en dos aspectos. En primer lugar, debido a la permanencia en su responsabilidad tradicional en las tareas domésticas y de reproducción, les exigió encontrar nuevas formas de acceso a los beneficios perdidos, a través del desarrollo de estrategias que les permitieran cubrir las necesidades básicas de todos los miembros del hogar con ingresos insuficientes y políticas sociales limitadas. En segundo lugar, el deterioro de los ingresos exigió a las mujeres una participación cada vez mayor en la fuerza laboral, manteniéndose casi sin modificaciones la desigualdad de género en lo que respecta al trabajo reproductivo (Wainerman, 2000), situación que se ha reflejado en trayectorias laborales femeninas discontinuadas.

Las consecuencias de las políticas de flexibilización laboral impactaron aún más negativamente en los hogares de las/os asalariadas/os agrícolas profundizando la inestabilidad y discontinuidad en las inserciones laborales en un sector que se ha caracterizado históricamente por la precariedad y la falta de regulación que se observa en la alta proporción de trabajo no registrado.

Este artículo analiza la participación de las mujeres en el trabajo asalariado agrícola en el Valle de Uco en la provincia de Mendoza, Argentina, a partir de su articulación con el trabajo doméstico y considerando las características de los hogares.

En este sentido, se llevo adelante el estudio de las inserciones laborales de las mujeres que se desempeñan como trabajadoras asalariadas en la agricultura del Valle de Uco en la provincia de Mendoza en la Argentina. Desde mediados de la década de 1990, dicha zona evidencia un notable proceso de reestructuración de la producción agrícola que ha tenido influencia en la organización del proceso de trabajo en los diversos cultivos que allí se producen. Se suma a esto una mayor participación de las mujeres, vinculada a la reestructuración mencionada, producto de la importancia otorgada a ciertas etapas del proceso de producción. Si bien, es importante destacar la presencia histórica de las mujeres en tareas agrícolas en esta zona, actualmente su trabajo adquiere mayor importancia a la luz de algunos requerimientos productivos específicos.

El presente artículo fue realizado en base a reiterados trabajos de campo efectuados durante tres años consecutivos en los departamentos de Tunuyán, 
Tupungato y San Carlos que conforman la zona conocida como Valle de Uco y se encuentran en el centro-oeste de la provincia de Mendoza. Se empleó una estrategia metodológica cualitativa basada en entrevistas a empleadores, tanto en empresas como en unidades productivas. Por otra parte, se entrevistó a trabajadoras asalariadas utilizando la historia de vida para reconstruir sus trayectorias laborales en el contexto de las etapas del ciclo vital atravesadas por ellas.

\section{Género y mercados de trabajo}

El análisis de la participación laboral desde la perspectiva de género y la división sexual del trabajo abre nuevas líneas de estudio que permiten dar cuenta de la complejidad que implican las inserciones laborales. En este sentido, las categorías de género y la perspectiva de la división sexual del trabajo permiten ir más allá de los determinantes económicos que, si bien definen aspectos centrales de la participación laboral, no explican estas inserciones laborales en su totalidad.

La categoría de género expresa la dimensión social de los roles sexuales; a la vez que son entendidos como construcciones históricas, observables a través de prácticas sociales que al repetirse y legitimarse delinean la participación de mujeres y varones en todos los aspectos de la vida social, incluso el trabajo. En el contexto de este enfoque, el concepto de división sexual del trabajo aporta una lectura sobre la dimensión sexuada de la participación laboral. Asimismo, como herramientas de análisis no solamente explican la relación entre varones y mujeres sino que además analizan las instituciones, símbolos y sistemas político-económicos en los que se producen y reproducen éstas prácticas sociales.

Este marco conceptual pone en un lugar central el rol social que asumen varones y mujeres para analizar la participación laboral. La asociación de los varones con la figura de principal proveedor económico del hogar y de las mujeres como principales responsables de las tareas reproductivas constituye una parte de la explicación sobre el tipo de puestos de trabajo asignados a cada sexo, como así también la duración de las contrataciones y, en el plano de la jerarquización de las tareas, lo referido a las diferencias salariales.

En esta línea, Roldán (1982) analiza la participación de las mujeres en el mercado de trabajo condicionada por el papel genérico adscrito dentro de la unidad doméstica. Como hijas, esposas-madres y/o jefas de hogar. Estas relaciones, a su vez, varían por la etapa del ciclo vital y la fase biocultural del grupo doméstico. Este enfoque puntualiza en las diferencias que se observan en las experiencias de los distintos subgrupos dentro del colectivo de trabajadoras asalariadas. Además, permite sistematizar las diferencias generacionales en las combinaciones de inserciones laborales por las que optan las trabajadoras para completar un ciclo anual de ocupación.

Por otra parte, a partir de los estudios feministas, sobre todo desde la década de 1970 (Borderías y Carrasco, 1994), se incluye en el concepto de trabajo a 
aquellas tareas reproductivas realizadas mayoritariamente por mujeres. Estas líneas de investigación ampliaron las dimensiones de análisis de la participación femenina en la fuerza laboral. En este sentido, los estudios no se limitan al empleo femenino sino que toman en cuenta las múltiples actividades realizadas por las mujeres que se superponen con el trabajo asalariado. En este marco, el concepto de división sexual del trabajo retoma las prácticas sociales específicas en cada sexo para dar cuenta de la participación diferencial de varones y mujeres en los ámbitos de la producción y la reproducción social. Además, estos enfoques plantean, que el trabajo femenino se desarrolla en una doble jornada laboral en la que se incluye el empleo y el trabajo reproductivo realizado por las trabajadoras. Asimismo la conceptualización sobre la doble jornada contiene aquellas actividades de articulación entre los dos espacios laborales, es decir la multiplicidad de arreglos vinculados a organizar y compatibilizar la jornada laboral asalariada con las tareas reproductivas y de cuidado de los miembros dependientes.

\section{Los mercados de trabajo agrícolas}

Las especificidades de la producción agrícola conforman uno de los factores que interviene en la construcción de inserciones laborales discontinuas a lo largo del año, influyendo en la precarización del trabajo en la agricultura. Puede mencionarse como característica de la actividad agrícola, la estacionalidad productiva y su consecuente discontinuidad en la demanda de mano de obra. Esta realidad conforma un marco particular para el desarrollo de los mercados de trabajo en esta actividad donde estacionalidad y discontinuidad se vinculan a formas de regulación social caracterizadas por contrataciones informales, bajos niveles de ingresos para los trabajadoras/es en general como también malas condiciones de trabajo, de transporte y de vivienda. (Fabio y Neiman, 2010)

Las trayectorias laborales en estos mercados de trabajo se distinguen por una marcada inestabilidad ocupacional a la que se suman formas de contratación que, por sus condiciones, impiden a las/os trabajadoras/es el acceso a beneficios contenidos en una relación salarial clásica tales como jubilación, aguinaldo, obra social y asignaciones familiares.

Para la mayor parte de las/os trabajadoras/es, el empleo en el sector agrícola queda supeditado a los momentos del ciclo productivo donde aumentan los requerimientos de mano de obra en tareas vinculadas al mantenimiento de los cultivos (podas, raleos, fumigaciones, aplicaciones de agroquímicos, etc.) y a las cosechas. Esta situación provoca la construcción de ciclos de empleo que requieren de la rotación de las/os trabajadoras/es por diferentes unidades productivas aportando a la profundización de la precariedad laboral en el sector. A este marco se suma una fuerte segmentación de la mano de obra en estos mercados de trabajo que restringe el acceso de determinados grupos de trabajadoras/es a las empresas que mejores salarios pagan y a las tareas mejor remuneradas (Piñeiro, 2008). Esto afecta, sobre todo, a la mano de obra femenina, particularmente en lo que respecta al acceso a tareas de mayor remuneración. 
En resumen, se trata de inserciones laborales discontinuas producto de la estacionalidad del sector a las que se suman una serie de normas tanto formales como informales que facilitan irregularidades en las contrataciones de trabajadoras/es. De la combinación entre la transitoriedad en el empleo y los bajos salarios percibidos a través de contrataciones, que en su mayoría no contemplan beneficios sociales, resultan condiciones de inestabilidad económica que afectan a las/os trabajadoras/es y sus familias. Estos mecanismos de precarización del trabajo agrícola se observan en la manera en que las/os trabajadoras/es llevan adelante sus vidas cotidianas alcanzando apenas condiciones mínimas que permiten su reproducción. En este sentido, algunos estudios han destacado que para comprender la situación de las/os trabajadoras/es asalariadas/os en la agricultura es necesario un análisis que incorpore los 'ámbitos reproductivos' y las formas de socialización por las que han sido atravesadas/os. (Benencia y Quaranta, 2006)

Más precisamente, Roldán (1982) señala que para el estudio de los mercados de trabajo agrícolas es muy importante considerar el carácter social que asumen los procesos de asalarización individual, puesto que no pueden interpretarse como reacciones individuales de quienes buscan vender su fuerza de trabajo sino como decisiones que son resultado de estrategias grupales de sobrevivencia.

A partir del estudio de la participación de las mujeres en el trabajo agrícola asalariado en paralelo con su desempeño en el trabajo reproductivo, es posible observar que el acceso restringido de las mujeres al mercado de trabajo agrícola tiene su correlato en las responsabilidades reproductivas socialmente asignadas a éstas.

\section{Mujeres asalariadas en la agricultura}

Diversos estudios sobre las características de la participación de las mujeres en los mercados de trabajo agrícolas abordaron las particularidades de las tareas en las que se desempeñan para comprender bajo qué condiciones se concreta esta presencia. Desde esta perspectiva, la organización del trabajo se basa en un esquema de división sexual de tareas para responder a los controles y exigencias externas sobre este tipo de producciones. Esta división de tareas se sostiene con la definición de cualidades femeninas, construidas socialmente, como "el sentido estético, la agudeza visual y la sensibilidad". (Bendini y Bonaccorsi, 1998) (Roldán, 1982; Lara, 1995; Bonaccorsi, 1998; Goldman, 1999; Candela y Piñon, 2004)

A la vez, estos estudios señalan que las mujeres ocupan los puestos más inestables y estacionales, en las tareas de menor remuneración. Además, no son reconocidas las calificaciones y competencias adquiridas por ellas en sus trayectorias laborales, aún cuando el mercado de trabajo exige de las mujeres amplias condiciones de "polivalencia" que les permiten desempeñarse en una variedad de puestos de trabajo en el proceso productivo. Por todo esto, en general la participación de las mujeres se limita a tareas que requieren 
habilidades manuales. (Cavalcanti, Ramos y Da Silva, 1998; Salamea y Waters, 1995; Vazquez Laba; 2007).

Como resultado, se observa que la división de tareas por sexo, dentro de los procesos productivos en la agricultura, no se asocia estrictamente a criterios técnicos sino que encuentra en estos esquemas una manera de organizar y jerarquizar la fuerza de trabajo sexualmente diferenciada. Es justamente en la jerarquización de las tareas donde se expresa la mayor desigualdad de oportunidades entre varones y mujeres, ya que allí la construcción social de las calificaciones juega un rol central (Lara, 1995) En este sentido, la asignación de puestos de trabajo a las mujeres se establece por la vía de la naturalización de ciertas habilidades y conocimientos que se suponen adquiridos en el ámbito doméstico; por ello las destrezas necesarias para el desempeño en tareas manuales no son consideradas calificaciones o aprendizajes obtenidos a través de las experiencias de las trabajadoras.

En definitiva, el proceso de segmentación de la mano de obra por sexo cumple un rol fundamental en las estrategias de acumulación de capital en el que las empresas apelan a determinados rasgos de género para organizar más eficientemente la mano de obra.

Los estudios sobre el empleo femenino en la agricultura, en el contexto de la reciente reconversión productiva, verifican la existencia de una organización del trabajo y una distribución de tareas fundamentada en la división sexual del trabajo. La reorientación de los sectores agrícola y agroindustrial hacia producciones no tradicionales, o bien la aplicación de nuevos parámetros productivos en las producciones tradicionales, demandó una especial atención a la etapas de cosecha, postcosecha y empaque que, desde las empresas, se organizó en gran medida con mano de obra femenina. (Lara, 1995).

En este sentido, no hay nada novedoso en las características de la participación de las mujeres en estos espacios productivos. Más bien lo que sucede es que las empresas han respondido a los requerimientos de modernización y de estrictos cuidados en la calidad con una organización del proceso de trabajo basada en la división genérica del trabajo y en los roles de género tradicionales socialmente difundidos.

Deere y León (1986) señalan que la participación laboral de las mujeres en estos espacios continúa vinculada al despliegue de habilidades manuales que se suponen naturales y por ello no se da lugar al reconocimiento de un aprendizaje producto de una trayectoria en los diferentes puestos de trabajo. Además, la participación en el trabajo asalariado es considerada, en estos contextos, secundaria al rol eminentemente femenino, es decir, al desempeño en los ámbitos reproductivos. Esto obtura las posibilidades de las trabajadoras para desempeñarse en puestos de trabajo de mayor continuidad durante el año y las sujeta a los períodos de alta demanda durante los picos del ciclo productivo. 


\section{Entre el trabajo y el hogar}

Las características temporarias del empleo, la inestabilidad en la percepción de los ingresos y los bajos salarios son las causas estructurales que llevan a las trabajadoras a la combinación de diferentes ocupaciones dentro y fuera de la agricultura y, a su vez, a la realización de diferentes tareas durante distintas épocas del año dentro del sector agrícola. El objetivo de estas combinaciones es lograr un ciclo ocupacional que garantice ingresos durante la mayor parte del año. Se trata de hogares de asalariados donde las fuentes de ingresos son casi exclusivamente provistas por el trabajo de sus miembros, contando con algunas otras provenientes de planes estatales de asistencia social, careciendo, en general, de algún tipo de producción doméstica de subsistencia. En algunos casos, cuando la relación con sus empleadores lo permite, pueden llevarse a sus casas algunos productos (tomates, ajo, pimiento, papas, etc.) con los que se elaboran conservas que se reservan para el invierno. En este contexto, la supervivencia se apoya en la combinación de los ingresos percibidos por los miembros del hogar que trabajan.

Para analizar los procesos de asalarización de las mujeres vinculados a las características de estos hogares, se ha tenido en cuenta la cantidad de miembros, la edad de los hijos, su conformación como nucleares o extensos y la etapa del ciclo vital en el que se encuentran. Entendemos que estas características condicionan las estrategias y las posibilidades de inserción laboral de las mujeres. Nos referimos a las elecciones que las trabajadoras realizan, dentro de un conjunto de alternativas disponibles, ya sea en función del armado de un ciclo de trabajo "constante" durante el año como de compatibilizar el trabajo dentro y fuera del hogar. En la conformación de estas estrategias, la edad, la posición dentro del hogar y la etapa del ciclo vital por la que atraviesan, actúan condicionando su desarrollo.

Con mayor frecuencia en los hogares nucleares que en los extensos, el cuidado de los hijos menores es uno de los motivos de la interrupción de las trayectorias laborales de las mujeres. El trabajo fuera del hogar es retomado cuando los hijos tienen edad suficiente para acompañar a las trabajadoras 0 bien cuando los mayores pueden cuidar a sus hermanos. Pero nuevamente, los bajos ingresos percibidos y la inestabilidad propia de la inserción en trabajos temporarios, hacen que la interrupción del trabajo extradoméstico de las mujeres impacte directamente en la percepción de ingresos de los hogares; es por ello que las mujeres interrumpen su inserción asalariada por períodos de tiempo lo más breve posibles. De todas formas, esta posibilidad se restringe a aquellos hogares que cuentan con otros miembros que pueden aportar ingresos.

En el caso de los hogares donde conviven varias familias o las/os hijas/os tiene edad suficiente, el cuidado de los menores se facilita a través de arreglos familiares de distinto tipo que, en general, son acordados y llevados adelante entre las mujeres. De esta forma, la organización del cuidado de los hijos y de las tareas domésticas "se reparte" entre las mujeres que lo habitan evitando la interrupción del trabajo asalariado. 
En el caso de Isabel (32 años) sus hijos más pequeños quedan a cargo de su hija mayor de quince años que, por decisión de la familia, se dedica a estudiar y no participa del trabajo asalariado.

Para Nidia, que tiene tres hijos, la inserción laboral a destajo le permite combinar las tareas productivas y reproductivas durante la jornada laboral mientras que a su vez destaca la importancia de buscar trabajo en las fincas cercanas a su casa. Trabajar en una finca cercana a su casa es importante para Nidia porque le permite regresar a mediodía a su casa: "llego al mediodía, vuelvo cocino y mientras se hace la comida voy haciendo las cosas de la casa y a la tarde me vuelvo a trabajar" Nidia comenta que para las tareas domésticas recibe una pequeña ayuda de sus hijos: "alguno se hace la cama pero nada más, el resto de la casa la hago yo".

Para el desarrollo de esta estrategia de inserción laboral Nidia considera fundamental el lugar donde reside, en este sentido afirma que es ventajoso para ella vivir en zona alejada de la localidad de "La Consulta": "acá se consigue más rápido trabajo está más cerca, acá es lejos para ir a estudiar y allá es lejos para venir a trabajar" Cuándo tiene que buscar trabajo Nidia se acerca a las fincas de la zona y habla con el encargado, son fincas que ella conoce porque hace más de veinte años que vive en la zona. Igualmente, Nidia remarca que no le importa demasiado conocer, con anterioridad la finca o el cuadrillero con el que va a trabajar: "Mientras traiga trabajo es lo mismo cualquier cosa para mí".

María (20 años) vive junto a su familia paterna y tiene un bebé de un año; en su caso, vivir con sus padres le facilita el cuidado de su hijo mientras ella trabaja. Es la madre quién se encarga del cuidado del bebé de María aunque a veces también es ayudada por su hermana de 17 años que trabaja fuera del hogar solamente durante el verano. María entiende esta ayuda de su madre como un hecho "natural" porque cuando su madre trabajaba fuera de la casa era ella quién se encargaba de sus hermanos: "Me ayuda a mi, antes la ayudaba yo".

Nancy (34 años) también relata que cuando sus hijos eran pequeños los dejaba al cuidado de su madre que vivía muy cerca de su casa. Como Nancy y su marido trabajaban todo el día en una finca alejada de su casa, los hijos quedaban todo el día con su abuela quién les preparaba la comida y se encargaba de llevarlos a la escuela.

Aunque las mujeres no estén casadas y no tengan hijos propios, su rol de hijas dentro del hogar influye en el desarrollo de su inserción laboral como asalariadas, puesto que por su condición de mujeres contribuyen al trabajo doméstico ayudando a sus madres o hermanas en el cuidado de los niños o en la realización del trabajo doméstico. Por ejemplo, María (20 años) comenta que finalmente dejó la escuela ya que no asistía asiduamente porque tenía que ocuparse de sus hermanos y de la casa cuando su madre trabajaba fuera del hogar.

Como señalamos, en la asalarización de las mujeres es importante también el rol que ocupan dentro de sus hogares. La condición de hija, esposa y madre o jefa de hogar va modificando los objetivos de la incorporación al mercado de 
trabajo y las formas de acceso al trabajo asalariado a lo largo de las trayectorias. Según el rol que las mujeres desempeñan en sus hogares, la percepción y los propósitos acerca de sus trabajos muestran algunas diferencias, sin olvidar que las condiciones socioeconómicas de los hogares determinan el margen de maniobra de estas elecciones.

Por ejemplo, algunas trabajadoras que han formado su propio hogar o tienen hijos eligen aquellas formas de contratación que les permiten regresar a sus casas al mediodía; este objetivo puede lograrse mediante una combinación entre una forma de contratación (en cuadrillas o individualmente) y la forma de pago (por jornal o por destajo). A su vez, a esto se suma la búsqueda de trabajo en fincas cercanas a sus casas.

Isabel trabaja a destajo junto con su marido. Durante la mañana trabajan desde las siete y media hasta las once. A las once se retiran de la finca y regresan a su casa; en este tiempo Isabel recibe a sus hijos y almuerza con ellos. A la una de la tarde salen nuevamente hacia su lugar de trabajo y se retiran a las cinco y media de la tarde. Manejarse con estos horarios y ritmos de trabajo es importante para Isabel porque de esta forma puede regresar a terminar de preparar el almuerzo para sus hijos. Finalizar la jornada laboral extradoméstica más temprano le permite disponer de tiempo para a realizar las tareas domésticas por la tarde. Isabel y su marido prefieren ir a los lugares de trabajo en sus propias bicicletas. Isabel comenta que no siempre es posible, pero es lo que prefieren: "Nosotros vamos en bicicleta porque terminas tus tareas y te vas, en cambio sino tenés que esperar al patrón que te traiga y son una vuelta por que llegas a las siete y media de la tarde de una tarea que terminaste a las cinco y media" Llegar a sus lugares de trabajo por sus propios medios es otro de los factores que le permiten a Isabel organizar su jornada laboral dentro y fuera de su hogar. Nidia también elige trabajar en fincas cercanas a su casa esto le permite regresar al mediodía y dedicarle tiempo a las tareas domésticas y al preparado de la comida para su familia.

Tanto Isabel como Nidia viven en hogares conformados por sus maridos y sus hijos y ninguna de las dos cuenta con familiares cercanos a quienes recurrir mientras están trabajando, por lo que el peso de las tareas domésticas recae casi exclusivamente en ellas. Isabel tiene 39 años y comenzó a trabajar en la agricultura a los trece años junto con su mamá; entre las dos trabajaban en "lo que viniera". Los dos hermanos mayores de Isabel también realizaban trabajos en la agricultura pero trabajaban junto con su padre. Comenta que trabajaban juntas con su mamá porque preferían el pago a destajo y no por día: "porque al tanto ganas más que lo que ganas al día, y eso es lo que a mi me gustaba con mi mamá". Además de ganar más dinero Isabel explica que la jornada de trabajo a destajo les permitía regresar a su casa y dedicarse al trabajo doméstico: "Así si vos tenías que venir a hacer algo en tu casa, a lavar, todo eso lo podías hacer porque salías más temprano y en cambio al día tenés que cumplir tus horas y al tanto no".

En el caso de los hogares extensos, donde otros integrantes pueden hacerse cargo del cuidado de los niños y las tareas domésticas, y de trabajadoras sin hijos, las mujeres tienen posibilidades de trabajar durante todo el día y en 
lugares más alejados de sus casas, ampliando así las posibilidades de conseguir trabajo.

Los objetivos de las inserciones laborales también muestran diferencias según la edad de las trabajadoras y su rol dentro del hogar; en muchos casos el trabajo asalariado de las mujeres es necesario para cubrir necesidades estructurales de sus hogares como comida y vestimenta. Igualmente es sumamente interesante que aún siendo así algunas de estas mujeres afirmen que sus ingresos representan una "ayuda" para el hogar: "y, una platita mas no viene mal".

Cuando se les pregunta a que destinan sus salarios sobre todo las mujeres que tienen hijos contestan que a la compra de ropa para los hijos pequeños o "sus cositas para escuela" o bien a alguna mejora en la casa y compra de muebles o electrodomésticos para el hogar. En el caso de las jefas de hogar que tienen hijos manifiestan que sus ingresos se destinan a la comida y al mantenimiento general de la casa.

La forma en que las mujeres, sobre todo las que están casadas, se refieren al destino de sus ingresos, aún cuando estos son centrales para la supervivencia del hogar, es similar a la que Stolen (2004) encuentra en su trabajo sobre una colonia de productores familiares algodoneros en la provincia de Santa Fe. En este trabajo la autora destaca que la manutención del hogar constituye un deber exclusivamente masculino, incluso la capacidad para mantener a la familia es directamente asociada con la masculinidad, pero a tal extremo que los varones que requieren ayuda económica de sus esposas no son considerados hombres "de verdad" (pp. 171). Respecto del destino de los ingresos adquiridos por las mujeres este estudio revela que, al igual que en los casos que analizamos, debe ser gastado en mejoras en la casa, muebles y ropa. De todas formas es importante contextualizar que los gastos considerados "extra" por las trabajadoras agrícolas de la zona de estudio muchas veces se destinan a mejoras estructurales en sus casas vinculadas a necesidades básicas de estos hogares.

\section{Los eventos vitales en la organización de las trayectorias laborales}

Las motivaciones y los objetivos que tienen las primeras inserciones laborales para las trabajadoras vuelven a mostrar variaciones según la edad y el rol desempeñado en el hogar. Los testimonios de las trabajadoras más jóvenes presentan la incorporación al trabajo como medio para alcanzar bienes materiales que juzgan propios para su edad.

De todas maneras, está presente en las valoraciones de las trabajadoras que el hecho de cubrir sus gastos individuales descarga de esa responsabilidad a los ingresos percibidos por sus madres y padres que se destinan exclusivamente a la compra de alimentos y al mantenimiento general de la vivienda. Esta valoración nos permite interpretar que si bien las motivaciones se presentan como objetivos individuales para alcanzar deseos personales "de una chica joven", las trabajadoras dan cuenta de que sus inserciones laborales 
tempranas constituyen aportes al presupuesto familiar. Por otro lado, es importante tener en cuenta que la intermitencia en las inserciones laborales, los bajos salarios percibidos y la generalizada precariedad en la relación laboral, presiona a las familias de los trabajadores/as para aumentar la cantidad de miembros insertos en el mercado de trabajo.

Marina tiene 18 años y comenzó a trabajar como asalariada agrícola a los quince. Empezó a trabajar porque el único ingreso fijo en el hogar era el de su padre; con esos ingresos se pagaban los impuestos de la casa y la comida para toda la familia y "no alcanzaba para los gustos de una chica joven". Con el objetivo de cubrir sus gastos y ahorrar dinero para utilizar durante el invierno, Marina comenzó a trabajar en las temporadas de verano, al finalizar la época de clases. La primera experiencia laboral de Marina en el trabajo agrícola fue antes de los quince años, aunque esporádicamente acompañaba, junto a su hermana, a su mamá algunos días en la tarea de atada de viña.

María tiene 20 años, es madre de un niño de un año y vive con sus padres, sus seis hermanos y su hijo; desde los 15 años trabaja como asalariada agrícola. Tomó la decisión de comenzar a trabajar porque el único ingreso estable de su hogar era el de su padre, aunque su mamá también trabajaba, pero sus ingresos eran esporádicos; por otro lado, comenzar a trabajar significaba, en palabras de María, "poder vestirme como yo quería"

Claudia (32 años) empezó a trabajar en la agricultura a los trece años, esa primera experiencia fue junto con su papá, su hermana y uno de sus hermanos varones. Comenta que iban los cuatro juntos a trabajar y que su papá les enseñó el trabajo agrícola. El padre de Claudia era empleado permanente en una finca que, según recuerda, les permitía a sus obreros llevar a trabajar a sus hijos para que puedan ganar un "sueldo aparte". A los hijos de los obreros: "nos pagaban un suelo mínimo, pero era de nosotros"

Analía tiene 24 años y empezó a trabajar junto con sus hermanos varones mayores a los 12 años. Su primer trabajo fue en la cosecha de ajo y fueron sus hermanos los que le enseñaron a realizar esa tarea. Dejo la escuela cuando terminó séptimo grado y empezó a trabajar. En ese momento eran once hermanos y trabajaban los dos mayores y la madre. El padre de Analía quedó discapacitado producto de un accidente laboral. Él se desempeñaba como tractorista en una finca de la zona y según el testimonio de Analía nunca recibió la indemnización correspondiente por el accidente sufrido en su trabajo. La madre de Analía dejó de trabajar cuando sus hijos mayores pudieron hacerlo.

Nidia (40 años) comenzó a trabajar a los siete años junto con su madre, sus cinco hermanos y su hermana menor: "la ayudábamos cuando tenía que atar y en todo lo que se hace a la viña y después todo lo que se hace a la cosecha" (el padre de Nidia era contratista de viña) Cuándo los hermanos mayores de Nidia se casaron, ella y su hermana quedaron trabajando en la finca junto con sus padres. Cuando Nidia tenía trece años comenzó a trabajar por su cuenta, fuera de la finca donde trabajaban sus padres: "Trabajé por ahí en la chacra plantando tomates, ajo, todas esas cosas de la chacra" Nidia dejó de trabajar junto a sus padres para obtener un ingreso propio "porque era plata aparte, por 
el contrato (de vid de su padre) les pagaban un salario fijo y con eso aparte uno trabajaba en la chacra y era ganancia para uno". Nidia comenta que con el nacimiento de sus hijos discontinuó sus trabajos fuera del hogar y trabajaba solamente durante la época de las cosechas. Durante el invierno el único ingreso del hogar era el que percibía su marido. En cuanto pudo dejar al mayor de sus hijos al cuidado de los menores retomó su trabajo durante el resto del año. En este caso también la trabajadora regresa al mediodía para poder encargarse de la comida y de las tareas domésticas.

Claudia (32 años), al igual que Nidia, dejó de trabajar cuando se casó y se dedicó al cuidado de sus hijos y al trabajo doméstico. Luego de su separación volvió a su casa paterna, junto con sus cuatro hijos y su condición de jefa de hogar hizo que volviera a retomar el trabajo asalariado en la agricultura aún cuando dos de sus hijos son muy pequeños todavía. Claudia paga a una persona que cuida a su hija menor (de seis meses) desde la mañana hasta el mediodía. Durante la tarde el cuidado de los más pequeños está a cargo de la hija mayor de 12 años. Vivir junto con su hermano, su cuñada y los hijos de ellos le facilita el cuidado de sus otros hijos, dos varones de ocho y cuatro años. Mientras Claudia y su cuñada trabajan, los niños más grandes se encargan de algunas tareas domésticas como el preparado de la comida al mediodía además de acompañarse entre ellos.

Yolanda (54 años) empezó a trabajar a los nueve años en un secadero de frutas. Fue a trabajar con unas primas y cuenta que en ese momento las empresas contrataban chicos para trabajar. Las tareas vinculadas al trabajo en la tierra las aprendió junto a su padre, contratista de viña, en las fincas vitícolas donde creció. Yolanda da mucha importancia en su relato a la facilidad con la que aprendió a hacer su trabajo a pesar de que nunca asistió a escuela, aunque le hubiera gustado. Pero siendo la hermana mayor comenta que su padre prefirió que trabajara: "Pero a mi papá cuando yo empecé a trabajar ya le gustó más que yo trabajara a que fuera a la escuela..." Era la mayor de once hermanos (seis mujeres y cinco varones) y ella y su padre eran los únicos sostenes del hogar.

El análisis muestra una temprana asalarización de las trabajadoras. Alrededor de los 12 años la mayor parte de las mujeres entrevistadas ya se había insertado al mercado de trabajo y percibido un salario "propio". Igualmente, describen su primer trabajo diciendo que fueron a "ayudar" a uno de sus padres o a algún pariente. Estos eventos se producen a edades más tempranas aún sobre todo en el relato de las trabajadoras de mayor edad.

La presencia de las mujeres en el mercado de trabajo depende más que la de los varones de los eventos vitales que ocurren en sus trayectorias de vida, y son tenidos en cuenta como una variable de peso a la hora de insertarse en ellos; es decir que el rol en el hogar y el desempeño de las tareas domésticas de reproducción inciden en sus posibilidades y en sus elecciones de inserción en el trabajo asalariado.

Las responsabilidades que la división genérica del trabajo les asigna en la esfera doméstica acompañan estos procesos de asalarización reforzando en 
las mujeres la idea de "obligación" de compatibilizar su trabajo en ambas esferas; esto se traduce en una estrategia de inserción laboral asalariada que debe incluir espacios para cumplir con las responsabilidades asignadas en el trabajo dentro y fuera del hogar.

En la vida cotidiana de las mujeres, el trabajo reproductivo y el trabajo asalariado se presentan como esferas diferentes pero que se piensan de manera simultánea; es decir que la organización del trabajo doméstico no puede resolverse sin tener en cuenta las demandas del trabajo asalariado y viceversa. Esta realidad las obliga a pensar y a organizar sus jornadas de trabajo de forma tal que un espacio no entre en contradicción con el otro; este objetivo es difícil de lograr por la diferencia de funcionamiento de ambos espacios. En este sentido, podemos decir que, en el caso de estas trabajadoras, compatibilizar el trabajo doméstico con el trabajo asalariado requiere de la articulación de dos instancias de organización diferentes pero que se llevan adelante en forma paralela.

\section{La organización del ciclo laboral: entre las tareas manuales y las demandas del hogar}

En cuanto a las diversas formas de organizar un ciclo de ocupación en la agricultura, Piñeiro (2008) señala que, desde el punto de vista de las/os trabajadoras/es, la respuesta a la estacionalidad de la demanda de mano de obra es el intento de construir un ciclo de ocupación anual, que define como "el conjunto de ocupaciones en que se desempeña un trabajador en un período de tiempo y espacio determinado" (Piñeiro, 2008; 59)

Entre las posibles opciones para organizar un ciclo de ocupacional anual, el autor distingue tres formas diferentes de construcción del mismo. De esta manera, encuentra trabajadores que no cambian de ocupación durante el año, considerando en este grupo, fundamentalmente, a los trabajadores permanentes. En segundo lugar distingue al grupo de trabajadores que si bien rotan por diferentes puestos de trabajo durante el ciclo productivo repiten este ciclo de ocupación todos los años, incluso accediendo a las distintas ocupaciones a través de los mismos "patrones". Esta forma de organización del ciclo laboral permite a los trabajadores obtener cierto grado de estabilidad, logrado no por la permanencia en una ocupación sino por la permanencia en un "recorrido ocupacional fijo y predeterminado". En el lado opuesto, encuentra a aquellos trabajadores que cambian de ocupación y de patrón continuamente a lo largo del ciclo productivo, siendo estos últimos los que registran períodos más largos de desocupación.

Esta situación se refleja en el Valle de Uco donde se presentan dos momentos del año bien marcados por las características de la demanda de mano de obra en cuanto a la cantidad y especificidad de las tareas. Por una parte, tenemos la temporada laboral de "verano" que, en la zona, se extiende desde el mes de diciembre con la cosecha de frutales de pepita, principalmente cerezas y duraznos, a la que le sigue la cosecha de otros frutales como peras, manzanas y ciruelas que culminan a mediados del mes de febrero. A ello se le suman las 
primeras variedades de uvas que continúan hasta mediados del mes de abril, momento en que finaliza la temporada de "tareas de verano" tal como se la conoce en la zona. Este es el momento del año donde se concentra la mayor demanda de mano de obra y las mayores posibilidades de inserción en los mercados de trabajo para la totalidad de las/os trabajadoras/es.

La diversidad de cultivos existente en la zona de estudio posibilita a las trabajadoras insertarse en diversas tareas a lo largo del año. De esta manera las trabajadoras rotan por tareas manuales en distintos cultivos y en diferentes épocas del año con el objeto de lograr un ciclo ocupacional que sea lo más extenso posible.

Por lo tanto, la construcción de los ciclos ocupacionales de las mujeres depende tanto de la demanda de tareas manuales en cada momento del año como también de las condiciones del hogar de cada una de las trabajadoras. En cuanto a la situación del hogar, son factores de suma importancia la cantidad y edad de los hijos, el rol que desempeñan las trabajadoras dentro de sus hogares y la cantidad de miembros del hogar que trabajan. Según como varíen estas condiciones hemos encontrado diversas combinaciones de inserciones laborales que muestran diferencias en las experiencias de los distintos subgrupos dentro del colectivo de trabajadoras asalariadas agrícolas. Por otra parte, el análisis de los roles de género ocupados en los hogares constituye un importante aporte para dar cuenta de las diferencias generacionales que muestran las distintas opciones tendientes a la elaboración de un ciclo de ocupación.

Analía tiene 24 años, no tiene hijos y vive con sus padres y dos de sus hermanos varones. Los padres de Analía no trabajan y entre ella y sus dos hermanos sostienen todos los gastos del hogar. Decidió trabajar como "temporaria de invierno" en una importante finca de la zona dedicada al cultivo de vid. Esta elección le permite "aprovechar el trabajo de verano porque acá en invierno no hay nada, para hombres sí que está la madera, pero para mujeres no. Por eso elegí temporaria de invierno para poder trabajar todo hasta enero y trabajo en otro trabajo hasta junio que tengo otros trabajos de verano y en junio de vuelta a... (menciona el nombre de la finca donde trabaja en invierno) (Analía 24 años).

La elección de esta trabajadora apunta a garantizarse seis meses de trabajo continuo entre junio y enero. Este período, especialmente entre junio y septiembre, coincide con el momento de mayor desocupación en el sector en lo que respecta a la demanda de trabajo femenino. De esta manera, mediante la inserción laboral en diferentes cultivos en el período de enero hasta abril, esta trabajadora aprovecha la alta demanda de trabajadoras/es correspondiente a la temporada de verano.

El "trabajo de verano" del que habla Analía consiste en ocuparse como trabajadora agrícola en las tareas que corresponden a la temporada de verano: "alguna cosecha de durazno, o cualquier fruta, o si no la uva que es febrero, marzo, o si no de empleada acá piden por la radio y te presentas". Ella consigue trabajos agrícolas a través de cuadrilleros que van a ofrecerle 
trabajos a su casa y junto con sus hermanos deciden quién tomará ese trabajo. De cualquier manera, prefiere presentarse como "particular", es decir, sin integrar una cuadrilla, en la finca donde sabe que están pidiendo personal: "voy y pregunto y si me dan voy en bicicleta"

Además del trabajo en la agricultura, Analía consigue algunos trabajos temporarios en comercios de la zona que hacen pedidos de empleados por radio, o bien en casas de familia, especialmente en el período entre abril y junio: "Tenés que aguantártela hasta que empiece la atada en (menciona la finca donde trabaja en invierno) son unos dos, tres meses que estás sin trabajo. Pero siempre hay algún trabajo"

Yolanda tiene 51 años y siete hijos (cinco mujeres y dos varones) Actualmente vive con su marido, su hijo varón que trabaja en la agricultura y su hija menor que no trabaja y se dedica a sus estudios. Desde hace tres años Yolanda trabaja en una importante finca vitícola de la zona como "temporaria de verano" en tareas exclusivamente vinculadas a la viticultura. Esto significa que trabaja por seis meses desde octubre, "cuando llega la brotación", con un contrato por seis meses, hasta el fin de temporada hacia finales de abril, momento en que termina la cosecha. Durante estos meses trabaja en turnos de 8 horas de lunes a viernes: "el día sábado no trabajamos y es el día del lavado, el planchado y la limpieza de la casa"

Para los meses de invierno, Yolanda trabaja para la otra finca vitícola donde realiza las tareas de atada de viña; este trabajo comienza a fines del mes de mayo y termina a principios del mes de agosto: "ahí descanso unos días y entro de nuevo en: (menciona la finca donde es temporaria de verano) "a principios de octubre".

El trabajo de atada de viña lo realiza en conjunto con su hija mayor, desde hace 6 años. Comenta por qué consiguió este trabajo: "Ellos (se refiere a los dueños de la finca) me conocen desde que soy jovencita, saben que vine desde San Rafael y antes he trabajado con ellos, me conocen desde hace años ya saben que yo les ato todos los años"

Isabel tiene 32 años, está casada y con seis hijos, logra trabajar en la agricultura durante casi todo el año. Junto con su marido rotan por diferentes cultivos y tareas en la zona dependiendo de la época del año y los requerimientos de mano de obra. Isabel realiza tareas hortícolas en el cultivo de tomate y en el cultivo de ajo. Trabaja también en el cultivo de vid, uno de sus trabajos preferidos: "La chacra (se refiere a los cultivos hortícolas) es muy pesada, en cambio la uva es un trabajo más lindo más liviano, yo prefiero trabajar en viña".

A partir de mediados del mes de diciembre Isabel y su marido se dedican al trabajo en la viticultura. Isabel comenta que en la vid trabajan hasta marzo, momento en que finaliza la cosecha. Luego de la cosecha de vid trabajan en el cultivo de poroto por espacio de un mes. Isabel comenta que las fincas de poroto se encuentran muy cerca de su casa y esto le permite el regreso a la 
hora del almuerzo. Al finalizar las tareas en este cultivo Isabel y su marido comienzan a trabajar en tareas de plantación de ajo.

Isabel comenta "que descansa" durante un mes y medio en el invierno porque su marido trabaja en tareas de limpieza de canales de riego para las cuales es contratado por la Dirección de Irrigación de la provincia. Comenta que en esta tarea no acompaña a su marido porque "a las mujeres no las necesitan, necesitan a los hombres". Luego de este período donde no hay trabajo para Isabel, comienza la temporada de plantación de ajo, donde el ciclo laboral de Isabel se reinicia. En este caso, las características del hogar de la trabajadora y la presencia de hijos pequeños aumentan su carga de trabajo en tareas reproductivas. Esta situación, similar a la de otras trabajadoras, hace que su estrategia de continuidad laboral consista en la rotación por diferentes cultivos tanto hortícolas como frutícolas. La alta rotación por diferentes cultivos es central en la estrategia laboral de Isabel puesto que elige la forma de pago a destajo para poder regresar a su casa a mediodía y encargarse de las tareas domésticas.

La construcción del ciclo laboral de Nidia consiste en ocuparse en las fincas cercanas a su casa en tareas a destajo durante la temporada de trabajo de verano. Durante el invierno no trabaja en la agricultura porque considera que no hay tareas para las mujeres. De todas maneras afirma que cuándo sus hijos crecieron comenzó a trabajar durante más tiempo en la agricultura, pero sostiene que no tiene trabajo durante todo el año: "estos años he trabajado más para el tiempo de la atada de la viña, pero en invierno no hay tanto trabajo para la mujer"

Es el esposo de Nidia quién logra una inserción anual en la agricultura "Mi marido trabajaba en invierno en la poda de frutales y en la plantación de ajo, a medida que le van saliendo los trabajos". Nidia comenta que su marido tiene trabajo en la agricultura durante todo el año porque desde que era joven conoce la tarea de poda tanto de plantas frutales como de viña.

Nidia comenta que durante el invierno, el dinero que gana su marido alcanza solo para la comida de la familia, igualmente manifiesta que desde que sus hijos mayores trabajan ha mejorado la situación de su hogar en el invierno: "ahora ya no es tanto porque los niños trabajan y se compran las cosas para ellos y con esos ingresos sus hijos compran las cosas para ellos". Los dos hijos mayores de Nidia comenzaron a trabajar cuándo terminaron la escuela primaria: "Salir a estudiar acá es difícil porque tenés que tener vehículo o vivir en otro lado".

Los casos presentados muestran la forma en que el rol genérico desempeñado en el hogar demarca las posibilidades de construir el ciclo ocupacional en la agricultura. El primer caso es un ejemplo del ciclo ocupacional de una joven trabajadora cuyo rol dentro de la unidad doméstica es el de hija. El segundo caso presenta el ciclo ocupacional de una trabajadora que desempeña el rol de esposa y madre dentro de su hogar pero sus hijos ya no dependen de los ingresos familiares. 
El hecho de no tener hijos aún o que estos ya no sean dependientes les permite organizar un ciclo de ocupación que implica mayores compromisos laborales en cuanto a la relación contractual, y a la vez les garantiza estabilidad de ingresos durante un máximo de seis meses. Esta posibilidad se ve dificultada cuando las trabajadoras tienen que desempeñar tareas reproductivas y de cuidado de miembros dependientes del hogar. En este sentido los dos últimos testimonios expresan la elección de inserciones laborales donde las trabajadoras rotan por diversas tareas y empleadores durante el ciclo productivo. Este tipo de inserciones son preferidas porque les permite tomar trabajos en fincas cercanas a sus hogares y en combinación con la forma de pago a destajo, posibilita la articulación con la realización de tareas reproductivas durante la jornada laboral.

\section{Reflexiones finales}

La doble participación de las mujeres, como asalariadas y como principales responsables de las tareas de cuidado y reproducción en sus hogares, les plantea un ejercicio constante de articulación entre dos espacios con lógicas diferentes. Este hecho impone, en la vida cotidiana de las mujeres, la necesidad de coordinar sus horarios y actividades para cumplir con los objetivos que les plantean ambos espacios, con sus particulares ritmos y requerimientos. Este pasaje constante del trabajo asalariado al trabajo en el hogar requiere de la toma de decisiones y la realización de elecciones a las que los trabajadores varones generalmente no están obligados.

Sumado a lo anterior, las características del empleo en la agricultura vinculadas a los bajos ingresos percibidos y a la inestabilidad de los mismos, como producto de la temporalidad del empleo, impiden a las trabajadoras y sus familias destinar parte de su presupuesto para delegar en otras personas las tareas reproductivas y de cuidado. Es en este sentido que durante la temporada de trabajo agrícola, sobre todo las mujeres, intensifican sus jornadas de trabajo para lograr compaginar las tareas productivas y reproductivas

Pensando en la articulación entre trabajo asalariado y trabajo doméstico, el concepto de "compaginación" de Lobato (2000) da cuenta de la serie de tareas realizadas por las mujeres que apuntan a organizar sus jornadas de trabajo. Nos referimos a una serie de responsabilidades que descansan en las mujeres vinculadas a la sincronización de horarios y resolución de problemas para atender a las diversas necesidades del hogar y sus miembros. De esta manera hemos observado que la vida de las mujeres asalariadas no termina en la "doble jornada laboral". Entre su trabajo doméstico y el trabajo asalariado media un esfuerzo adicional dedicado a la organización de la vida cotidiana de todos los miembros del hogar y a las tareas de reproducción necesarias. Dicho esfuerzo finalmente les hace posible trabajar fuera de sus hogares.

Además, las tareas vinculadas a la organización del hogar y al cuidado y atención de las necesidades de sus miembros, se apoyan, en muchos casos, en la construcción de relaciones de reciprocidad entre familiares y vecinas que 
les permiten a las trabajadoras sostener su presencia en ambos espacios. Tanto la construcción como el funcionamiento de estas "redes de ayuda" se basan casi exclusivamente en acuerdos realizados entre mujeres. Este hecho muestra tanto la sobrecarga de trabajo en las mujeres como también la naturalización de la responsabilidad femenina en la atención del hogar y sus miembros.

Si bien es importante destacar la presencia histórica de las mujeres en tareas agrícolas en el Valle de Uco, el trabajo de las mujeres ha adquirido mayor importancia a la luz de requerimientos específicos vinculados a la reestructuración de la producción. Sin embargo, esta realidad no revela una mayor valorización social del trabajo femenino en la zona ni tampoco el reconocimiento de saberes adquiridos a lo largo de varias temporadas de trabajo que lleven a jerarquizar las tareas desempeñadas por las trabajadoras. En la zona de estudio, la participación femenina continúa interpretándose como secundaria en relación al trabajo masculino y asociada a la naturalización de los roles de género. Por otra parte, estas representaciones del trabajo femenino limitan la participación de las mujeres a determinadas tareas impidiéndoles establecer un ciclo laboral más prolongado y el acceso a tareas de mayor jerarquía y remuneración.

\section{Bibliografía}

(1998) BENDINI, Mónica y BONACCORSI, Nélida, Con las puras manos. Mujer y trabajo en regiones frutícolas de exportación, Cuadernos del GESA, Ed. La Colmena, Buenos Aires.

(2006) BENENCIA, Roberto y QUARANTA, Germán, "Mercado de trabajo y relaciones sociales: la conformación de trabajadores agrícolas vulnerables" Sociología del Trabajo, nueva época, núm.58 p. 83-113.

(1987) BENERÍA Lourdes y ROLDÁN, Martha, "The Crossroads of class and gender. Industrial homework, subcontracting and household dynamics in México city.", Ed. University of Chicago press, Chicago.

(1998) BONACCORSI, Nélida "Ser embaladora de la fruta: una trayectoria de trabajo femenino" en Bendini, Mónica y Bonaccorsi, Nélida (comp.), Con la puras manos: mujer y trabajo en regiones frutícolas de exportación, Cuadernos del GESA, Ed. La Colmena, Buenos Aires.

(2003) CARRASCO, Cristina. "La sostenibilidad de la vida humana": ¿Un asunto de mujeres? en León, Magdalena (comp.), Mujeres y trabajo: cambios impostergables, REMTE, Porto Alegre, p 11- 49.

(1998) CAVALCANTI, Salete., RAMOS, Juliana y BELO DA SILVA, Ana, "EI trabajo femenino en la agricultura de exportación: Las trabajadoras en la producción de uva-Brasil (Valle de San Francisco) en Bendini, Mónica y Bonaccorsi, Nélida (comp.), Con la puras manos: mujer y trabajo en regiones frutícolas de exportación, Cuadernos del GESA,Editorial La Colmena, Buenos Aires, p77-91.

(2006) DEERE, Diana. "¿La feminización de la agricultura? Asalariadas, campesinas y reestructuración económica en la América Latina rural”. Ponencia magistral al VII Congreso de ALASRU, Quito, Ecuador. 
(1986) DEERE, Diana "La mujer rural y la producción en la periferia capitalista" en Magdalena León (ed.), Las trabajadoras del agro: debate sobre la mujer en América Latina y el Caribe, vol.2, Asociación colombiana para el estudio de la población, Bogotá.

(1999) GOLDMAN, Julie: "Interpretaciones de la producción agroindustrial: el control de la calidad en la industria frutícola chilena", en Grammont, Hubert y otros (coord.), Agricultura de exportación en tiempos de globalización. El caso de las hortalizas, frutas y flores, CIESAS-Juan Pablos Editor, México.

(1995) LARA FLORES, Sara, "La feminización del trabajo asalariado en los cultivos de exportación no tradicionales en América Latina: efectos de una flexibilidad salvaje", en Sara María Lara Flores (coordinadora), Jornaleras temporeras y bóias frias, UNRISID Editorial Nueva Sociedad, Venezuela.

(2000) LOBATO, Mirta, "Lenguaje laboral y de género en el trabajo industrial. Primera mitad del siglo XX" en Gil Lozano, Fernanda; Pita, Valeria e Ini, Gabriela (coord.), Historia de las mujeres en la Argentina, vol. II, Taurus, Buenos Aires

(2008) PIÑEIRO, Diego, El trabajo precario en el campo uruguayo, Ed. Universidad de la República, Montevideo.

(1982) ROLDÁN, Martha, "Subordinación genérica y proletarización rural: un estudio de caso en el noreste mexicano", en Magdalena León (ed.), Las trabajadoras del agro: debate sobre la mujer en América Latina y el Caribe, vol.2, Ed. Asociación colombiana para el estudio de la población, Bogotá.

(1995) SALAMEA, Lucía y WATERS, William, "La cuestión de género en la reestructuración de la agricultura ecuatoriana" en Lara Flores, Sara (coordinadora), Jornaleras temporeras y bóias frias, UNRISID Editorial Nueva Sociedad, Venezuela p 35-49

(2004) STOLEN, Kristi Anne, La decencia de la desigualdad: Género y poder en el campo argentino, Antropofagia, Buenos Aires.

(2007) VAZQUEZ LABA, Vanesa, Desorganizando la tradicional división sexual del trabajo familiar: un estudio comparativo de familias asalariadas rurales del Noroeste Argentino, Tesis Doctoral, Facultad de Ciencias Sociales, UBA (1986) WILSON, Fiona "La mujer y las transformaciones agrarias en América Latina: Revisión de algunos conceptos que fundamentan la investigación" en León, Magdalena y Deere, Carmen Diana (comp.), La mujer y la política agraria en América Latina, ed.Siglo XXI ACEP, Bogotá.

(2000) WAINNERMAN, Catalina, "División del trabajo en familias de dos proveedores. Relato desde ambos géneros y dos generaciones" en Estudios demográficos y urbanos, Vol 15, $n^{\circ} 1$. 